\title{
10 Years of Surface-Enhanced Raman Spectroscopy in Art and Archaeology.
}

\author{
Marco Leona ${ }^{1}$, Pablo S. Londero ${ }^{1}$ and John R. Lombardi ${ }^{2}$ \\ 1. Department of Scientific Research, The Metropolitan Museum of art, 1000 fifth Avenue, New York, \\ NY 10028, USA. \\ 2. Department of Chemistry, The City College of New York, 138th Street at Convent Avenue, New \\ York, NY 10031, USA
}

The identification of organic colorants in works of art has traditionally been one of the most difficult challenges in cultural heritage science. While non-invasive methods such as X-Ray fluorescence spectrometry (XRF), or Raman microscopy are extremely useful for the identification of inorganic pigments, they remain generally unsuitable for the analysis of most organic colorants. The absence of characteristic elemental markers renders XRF essentially useless (with notable exceptions, such as bromine in dibromo indigo - tyrian purple -), and the fluorescence of most organic dyes under laser excitation tends to result in poor Raman spectra.

In recent year Surface-enhanced Raman scattering (SERS) has emerged as an invaluable microanalytical technique for studies involving dyes and organic pigments. The applicability of SERS to the problem of detecting low concentrations of organic colorants on textiles fibers was demonstrated by Guineau [1], but it is only ten years ago that serious concentrated efforts to make SERS into a robust method suitable to museum practice began $[2,3,4]$. SERS is now an established technique in the field, and it could be argued that with the exception of immuno-SERS biomedical assays, the analysis of organic colorants and dyes in cultural heritage material is the principal practical application of SERS today.

Several groups are currently active in SERS research on cultural heritage, and considerable progress has been made in the study of natural dyes, and in the development of plasmonic substrates and analytical protocols [5]. Methods involving gels as sampling aids or as vehicles for silver nanoparticles have been proposed as a way to conduct quasi-non-invasive analysis $[6,7,8]$, inkjet systems have been developed to precisely deliver silver colloid nanodroplets directly on the objects to be analyzed [9], and a laser ablation coupled SERS technique has been shown to yield micrometer scale spatial resolution for SERS analysis [10].

Concerns over the difficulty in preparing reliable and stable supports, and the perceived difficulty in searching SERS spectra against digital libraries continue to slow down the diffusion of the technique. While several plasmonic substrates and sample treatment approaches have been employed in the study of dyes and colorants, we have found that the use of resonant excitation, a stable and highly efficient silver colloid [11], and a two-step measurement approach (analysis of the sample as-is, followed by recovery of the sample and a second analysis after a lossless non-extractive hydrolysis sample treatment [12] give the best results. Spectra thus obtained can be reliably compared with reference spectra, and searched against a SERS spectral library containing over one hundred spectra representing different dyes measured in different conditions [13]. While dyes differ widely in their SERS efficiency (a term used here to include both differences in SERS cross-section and the affinity of a dye for a given plasmonic substrate), thus complicating quantitative estimations of dyestuff components and sometimes making it impossible to detect the target analyte over matrix interferences, it can be shown that SERS is 
otherwise reliable and reproducible, and that SERS spectra can easily be compared with appropriate library references.

At the Metropolitan Museum of Art, SERS has been used to identify organic dyes in samples from over one hundred works of art, ranging in dates from $2000 \mathrm{BC}$ to the present. The range of dyes studied and identified in works of art includes madder, kermes, lac, cochineal, methyl violet, Nile blue and eosine, from Ancient Egypt to the Impressionists and to Contemporary Art. In addition, almost all natural dyes have been characterized by SERS, although some important classes, such as the flavonoids, remain difficult to identify in microsamples from ancient objects.

[1] B. Guineau, and V. Guichard, ICOM Committee for Conservation: 8th triennial meeting, Sydney, Australia, 6-11 September, 1987. Preprints.; The Getty Conservation Institute: Marina del Rey: 1987; Vol. II, p. 659-666.

[2] I.T. Shadi, et al, J. Raman Spectrosc. 2004, 35, 800-807

[3] M.V. Cañamares et al, J. Raman Spectrosc. 2004, 35, 921-927

[4] M. Leona, 6th IRUG Meeting, Florence, March 29- April 1, 2004.Proceedings; Il Prato, Padova: 2005; 105-112.

[5] F. Casadio et al, Accounts of Chemical Research 43(6): 782-791 (2010).

[6] Marco Leona et al, Anal. Chem., 2011, 83 (11), p 3990-3993

[7] B. Doherty et al, J. Raman Spectrosc. 2011, 42 (11), p. 1932-1938

[8] C. Lofrumento et al, J. Raman Spectrosc. 2013, 44 (1), p. 47-54, 2013

[9] D.P. Benedetti et al, Journal of Raman Spectroscopy 2014, 45 (1), p. 123-127

[10] P.S. Londero, J.R. Lombardi, and M. Leona, Anal. Chem. 2013, 85 (11), pp 5463-5467

[11] M. Leona, PNAS 2009, 106 (35) 14757.

[12] F. Pozzi et al, Anal. Chem. 2012, 84 (8), 3751.

[13] F Pozzi et al, Anal. Methods, 2013, 5 (16), 4205 - 4212 